\title{
Open access system of life quality management on the basis of spatial modelling of pollution in the coastal areas
}

\author{
Vyacheslav Vishnevetskiy ${ }^{1, *}$, Vladislav Popruzhniy ${ }^{2}$ \\ ${ }^{1}$ Southern Federal University, Institute of Nanotechnology, Electronics and Engineering, Taganrog, Russia \\ ${ }^{2}$ FGU Azovmorinformcenter, Taganrog, Russia
}

\begin{abstract}
The quality of human life consists of many parameters: physical, psychological, level of independence, social life and the environment. The latter, in turn, includes safety, ecology, security, availability and quality of medical care, information, training opportunities, and life. The system of life quality evaluation, combining an objective component (indicators of water and air quality, morbidity) and subjective (the degree of satisfaction of residents with water quality) was developed. "Crowd survey" data block realizes the function of "Open access". The system of life comfort includes information on people morbidity and the main consumed water and air parameters that affect the satisfaction with natural resources and health. Such system units as «Input Interface» and «Interaction Interface» have been developed as a mobile application. In addition, system includes geographic information system that allows visualizing, analyzing and predicting the state of the object. Thematic maps of spatial pollution of the Taganrog Bay at control points were simulated. The main features of the system are shown and recommendations for its application are offered.
\end{abstract}

\section{Introduction}

The economic and social development of human society takes place in conjunction with the production, use and emission of an ever-increasing number of chemicals $[1,2]$. Currently, about one hundred thousand chemicals are used every day. Their comprehensive monitoring together with a huge number of by-products released by chemical compounds is very difficult to conduct, and sometimes even impossible.

Although the ecosystem and humans constantly interact through complex compounds, only a few pollutants present in the environment have adverse effects on the living organism, causing various diseases, increasing the mortality rate [3-5]. Identifying these and many other pollutants is a key to controlling and improving the comfort of people's lives and reducing the risks of adverse impacts on biological sites.

The increasing scale of human influence on the environment, cause acute problems associated with its condition [6-8]. Polluted air has a negative impact on human health, increases animal mortality, depresses vegetation, so the assessment of the state of the air is relevant [9-12]. Monitoring of air pollutants is necessary to understand their spatial and temporal distribution and, ultimately, to minimize their harmful effects. When assessing the state of the air environment, one of the main tasks is to quantify the composition of the analyzed air [13-16].

Thus, the relevance of the research topic is determined by the need for a system by which, first, will be able to appreciate the comfort of life that allow us to monitor the anthropogenic impact on the hydrosphere, and secondly, it will be possible to assess the quality of water and air that will allow us to monitor comfort life.

Taking into account the above, the purpose of the work is to develop system of estimation of life comfort on the basis of theoretical analysis of data and results of own research data.

\section{Methods}

In many areas of science and technology, arise problems, the solution of which requires a comprehensive study of the interaction of technical means and a living organism. Such tasks include the creation of biotechnical technologies that include the impact of environmental factors on biological objects at the level of the organism as a whole. The study of biotechnical interaction and synthesis of biotechnical systems is possible on the basis of a unified description of the behavior of interrelated biological and technical objects from the standpoint of system analysis, the theory of complex systems and applied biology. In the development of modern biomedical engineering and bionics, there is a tendency to move from the study of individual biological systems to the study of complex synergetic systems in order to identify adequate methods of coupling biological objects with technical devices into single biotechnical systems, the main distinguishing feature of which is their pronounced emergence.

\footnotetext{
* Corresponding author: vuvishnevetsky@mail.ru
} 


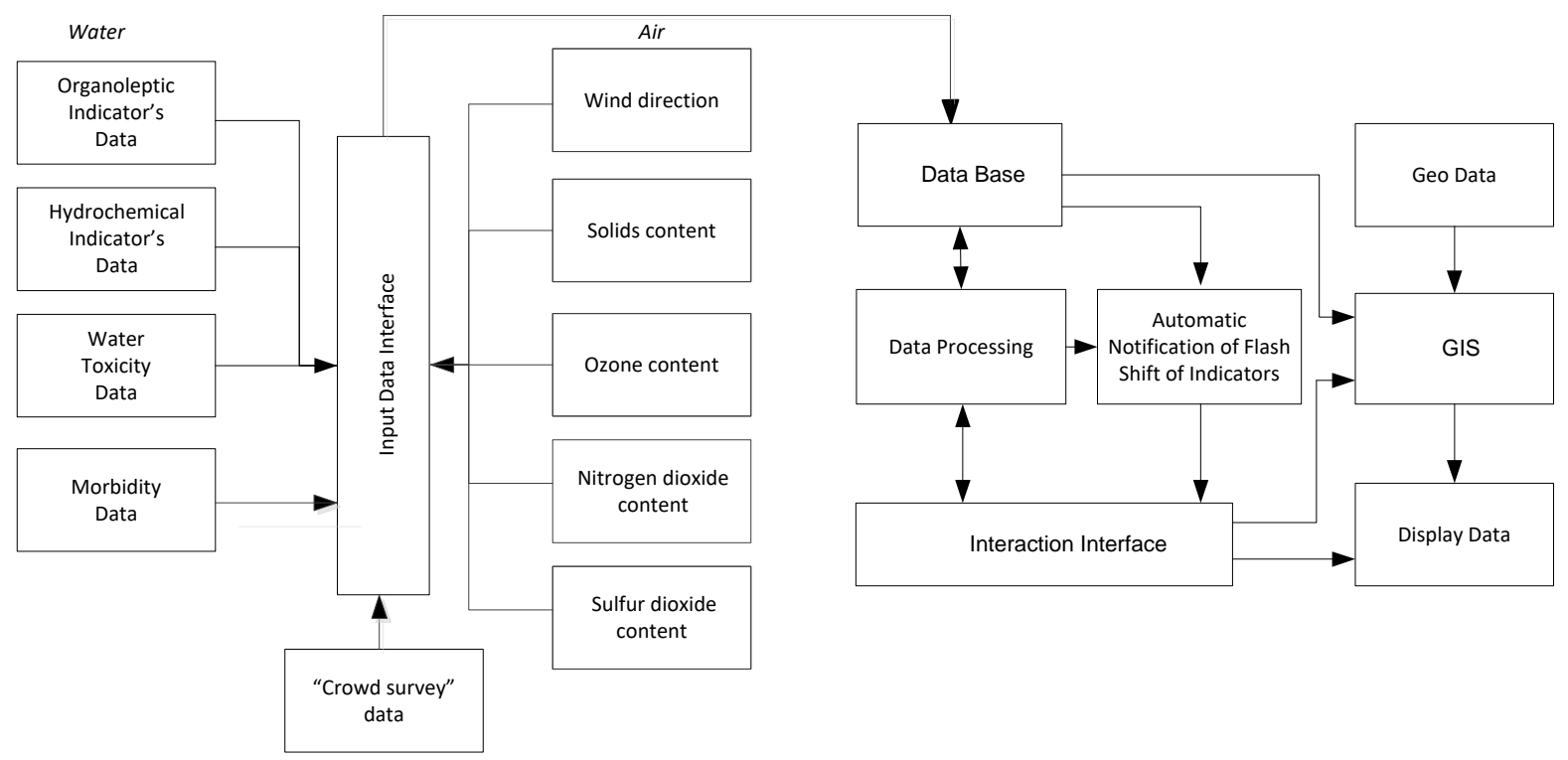

Fig. 1. Block-scheme of open access system of life comfort management.

Figure 1 shows the scheme of the system for assessing the comfort of life in relation to water quality data. This scheme was constructed on principles describe above. It consists of a group of software modules that are interconnected and united by a single interface.

Database is needed for the system's operation, which contain primary information. For example, for water such parameters as permissible concentration of pollutant, calculations of water pollution index, water quality classes, toxicity classes. In comparison to earlier designed scheme [8] new block of input data was added to the system - air parameters, according to recommendations of World Health Organization "Air quality guidelines”, 2005, [17]: contents of solids, ozone, $\mathrm{NO}_{2}, \mathrm{SO}_{2}$. Also we added wind direction, because the direction and speed of wind plays an important role in the spread of pollutants in the air [18], so this factor should be seriously considered in urban planning when planning settlements and the location of industrial enterprises and residential areas [19,20]. However, it should be taken into account that during the year the winds blow in all directions, so the environment is polluted in all directions to a greater or lesser extent.

One more new block appeared in the scheme "Crowd survey" data, that allowed to organize "Open access” mode. New information technologies have attracted thousands of people around the world to participate in the processes of acquiring new knowledge on a distributed informal basis. It is already recognized that such projects also contribute to formal science. New and unexpected ways of interaction between formal science and civil society have also emerged. Voluntary participation in knowledge creation is not a new phenomenon, but it has received an additional impetus with the advent of modern it. Scientific crowdsourcing and citizen science, which grew out of crowdsourcing projects, is a research practice in which the public (nonprofessional scientists) collaborate with professional scientists in conducting scientific research. Citizen science projects that use modern information technology are also known as "citizen cyber science". Similar projects for citizens in online versions have already shown their effectiveness in terms of obtaining new knowledge [21,22].

The assessment of comfort will be calculated as the average value of the normalized indicators of water and air quality, their toxicity, the level of morbidity and the results of the "crowd survey" on satisfaction with the quality of water and air resources.

Spatial modeling of pollution was carried out in the software complex MapInfo. MapInfo Professional is a geographic information system (GIS) that is designed to collect, store, display, edit, and analyze spatial data.

\section{Results and Discussion}

During the study, we applied the methods described above to construct thematic maps of spatial pollution of the Taganrog Bay at control points. They were created with the help of MapInfo software on the basis of averaged environmental monitoring data for 2007, 2014 and 2016 years, taken from the dynamics of pollution (Fig. 2). Gradation of water quality according to the pollution degree in accordance with the water pollution index is following: up to 0.2 - very clean (I quality class); 0.2-1.0 - clean (II quality class); 1.0-2.0 moderately polluted (III quality class); $2.0-4.0$ - polluted (IV quality class); 4.0-6.0 - dirty (V quality class); 6.010.0 - very dirty (VI quality class); $>10.0$ - extremely dirty (VII quality class).

According to Fig. 2, it is clearly seen that the water in the Taganrog Bay in 2007 belonged to class II quality and was "clean" at all sampling points. But over time (2014 and 2016) the condition of the waters deteriorated and became class III quality.

The developed system in a form of mobile application is aimed to analyze the data of environmental monitoring and "crowd survey" to assess the level of life 


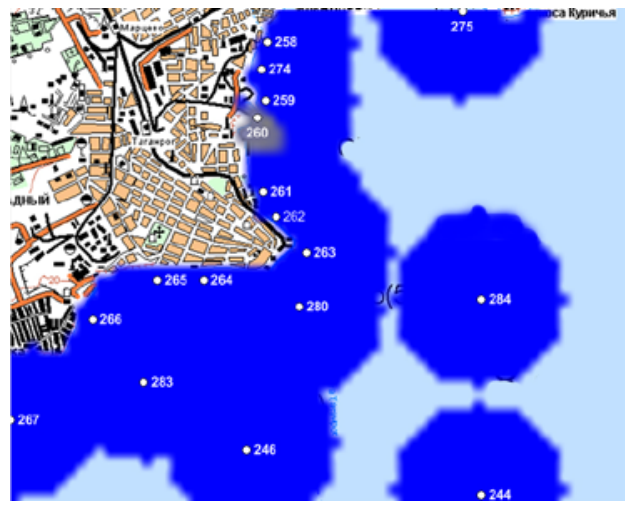

a)

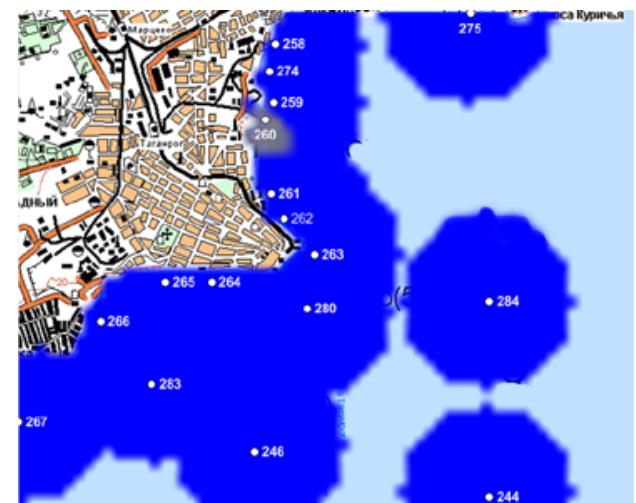

b)

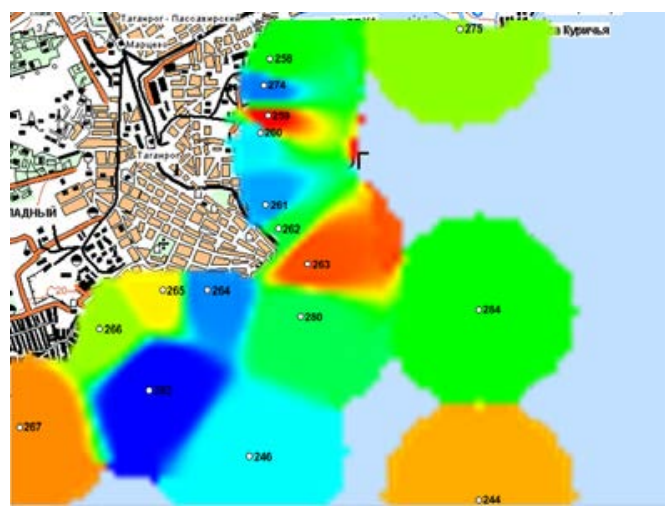

c)

Fig. 2. Spatial modeling of water quality changes in the Taganrog Bay by water pollution index for different years: a - 2007, b 2014, c -2016 . Colored areas correspond to different water pollution index: red -1.76 , yellow -1.6 , green -1.44 , blue -1.2 , dark blue -1.02 .

comfort, which will allow to timely identify and predict the development of negative processes that affect the quality of water and human health on the basis of input data. As a result, thanks to the data obtained, it will be possible to develop and implement measures to prevent the negative consequences of such processes.

In addition, the analysis of the survey data of people will create a general portrait of satisfaction with the quality of life from the point of view of individuals and assess the level of "public mood", which in turn affects the performance of people and their emotional state.

\section{Conclusions}

Thus, the system of assessing the comfort of life of the population can act as an auxiliary tool for assessing the effectiveness of management at the municipal level and determine the social development of the region, which is a balanced development of all spheres of life.

The authors plan in the future to add to the system data on the correlation of air quality and morbidity, obtained with the help of volunteers and to finalize the mobile application.

This publication was sponsored by Southern Federal University. The authors express their gratitude to Professor Irina Starchenko for scientific guidance and valuable advice.

\section{References}

1. A. Shaw and T. Saharan, Environment and Urbanization, 31(2), 597-614 (2019).

2. J. Vanderleeuw and J. Sides, Journal of Urban Affairs, 38(5), 661-675 (2016).

3. L.A. Catling, I. Abubakar, I.R. Lake, L. Swift and P.R. Hunter, J. Water Health 6(4) 433-442 (2008).

4. R. Chowdhury, A. Ramond, L.M. O'Keeffe et al, Clinical research ed. 362 k3310 (2018).

5. R. Cui, H. Iso, E. Eshak, K. Maruyama and A. Tamakoshi, Public Health Nutrition 21(16) 30113017 (2018).

6. S. Monarca, I. Zerbini, C. Simonati and U. Gelatti, Ann Ig. 15(1) 41-56 (2003).

7. K. Rehman, F. Fatima, I. Waheed and M. Akash, J. Cell Biochem. 119, 157-184 (2018).

8. V. Vishnevetskiy and V. Popruzhniy, Scientific Review Engineering and Environmental Sciences 28(1) 49-60 (2019).

9. A. Kumar, New Perspectives Quarterly, 36(2), 2123 (2019).

10. S. Rajagopalan, S. Al-Kindi and R. Brook, Journal of the American College of Cardiology, 72(17), A1-A30 (2018).

11. J. Argacha, European Heart Journal, 38(3), 141141 (2017). 
12. L. Bai, J. Wang, X. Ma and H. Lu, International Journal of Environmental Research and Public Health, 15(4), 780. (2018).

13. O. Raaschou-Nielsen, R. Beelen, et al, Environment International, 87, 66-73 (2016).

14. D. Costa, Toxicological Sciences, AdvanceArticle (1), 1-8 (2018).

15. J. Keller, T. Larson, et al, Environmental Epidemiology, 2(3): e024. (2018).

16. Z. An, Y. Jin, J. Li, W. Li, and W. Wu, Current Allergy and Asthma Reports, 18(3), 1-7 (2018).

17. WHO Air quality guidelines for particulate matter, ozone, nitrogen dioxide and sulfur dioxide Global update 2005 - Summary of risk assessment (2005).

18. L. Zhao and T. Haruyama, The World Economy, 40(8), 1646-1666 (2017).

19. S. Perry, D. Heist, L. Brouwer, E. Monbureau and L. Brixey, Atmospheric Environment, 142, 286-295 (2016).

20. D. Guo, P. Zhao, R. Yao, Y. Li, J. Hu and D. Fan, Journal of Applied Meteorology and Climatology, preprint (2019).

21. B. Young, N. Dodge, P. Hunt, M. Ormes, M. Schlesinger and $\mathrm{H}$. Shaw, Biological Conservation, 237, 57-62(2019).

22. M.-L. Schläppy, J. Loder, J. Salmond, A. Lea, A. Dean and C. Roelfsema, Frontiers in marine science. 1-7 (2017). 